\title{
Quantitative Image Analysis
}

Prof. dr. Vito Smolej, Carl Zeiss Vision, Zeppelinstraße 4, 85399 Munich- Halbergmoos, Germany

\section{Summary}

In a lot of cases a two-dimensional, flat image can be seen as a question posed to the person observing it. In a case of a police photo, we may want to find out the car model and its license plate number. When it is a ceramic sample under the microscope, it may be the porosity of the sample. And in a case of a patient biopsy, we may look for the proportion of cancerous cells in the tissue. And finally, allowing ourselves the jump into three dimensions, it may be the roughness of a polished surface or some specifics of the processes within a living cell.

All of these examples have the following two aspects in common:

- The answer to the question is quantifiable: it can be expressed as number or as a sentence, generally speaking as a file - a rather short file at that, compared to the original amount of information

- The answer is encoded one way or another in the image - at least there is a strong probability (sometimes accompanied by a strong hope...), that the information sought is there

For industrial-level, routine applications dependable automatic decoding methods are needed to get the answer from the image (actually the major part of the problem is finding the corresponding decoding operator). The approaches, algorithms and solutions, that address this problem, constitute the field of quantitative image analysis.

The process of quantitative image analysis is a stepwise process, with each step shedding a significant amount of irrelevant information from the image and thus increasing the proportion of the information relevant to the question posed. Here's an example of stepwise information reduction, typical for 2D images:

\begin{tabular}{llr}
\hline processing step & creates... & typical size (kB) \\
\hline image acquisition & gray scale image & 1.000 \\
gray scale image processing & gray scale image & 500 \\
discrimination & binary image & 64 \\
binary image processing & binary image & 32 \\
measurement & data file & 2 \\
\hline
\end{tabular}

Some of the new developments and advances in the steps, laid out above, will be addressed in the lecture. 
Image acquisition: Given the fact, that the information we are after must be in the original image in the first place, the questions of input devices (digital cameras, digital microscopes etc), their lateral and gray-level resolution and the necessary image size will be addressed. Additional interesting perspectives open up with possibilities of obtaining and analyzing $2 \mathrm{D}$ images, that are essentially unlimited in size. The 3D volume imaging, as well as the topics of the extended depth of focus and surface topography imaging, will also be addressed.

Gray image processing: Image processing is sometimes confounded with the broader term of image analysis. It includes a range of procedures that both use and produce gray level images. Their function is to stress some type of feature or information in the picture and destroy some other information that is of no practical interest. The same group of functions can of course be used also in three dimensions, for instance to deconvolute the acquired stack of images.

Segmentation: The discrimination step converts a gray level image into a binary image. The most common way of converting gray scale image to binary image is by discrimination or threshold, where a range of brightness that corresponds to features of interest is selected. Additional to gray-level segmentation there exist possibilities to use image attributes other than the gray level: local texture, orientation or local frequency in the image, edges between features etc.

In case of multidimensional images, such as deconvoluted reconstructions, this step allows us to separate the interesting part of the scene from the uninteresting 3D-background.

Binary image processing: binary images may need some further work before the measurement step is in place. There is a broad class of operators of "mathematical morphology" that smooth the outlines of features, connect or separate neighbors, isolate particular shapes etc

Image measurement: Eventually numerical data can be extracted from the image. The quantitative data may fall initially in two categories: the measurement may be global and correspond to the entire field of view, or it may apply individually to each object in the image. The former type of measurement includes such features as area percentage, mean chord length, or deduced parameters like volume percentage and specific surface. The latter measurement method provides information on sizes, shapes, positions and gray levels of objects.

Given the multitude of different parameters possible the question will be asked and also answered: "What does it mean - everything?" A ranking of most popular parameters will be of interest as well.

Data post processing: with a number of objects of same type on hand, it is natural to try to synthesize the information produced in some useful fashion, for instance in a form of a statistics report or as plot of a distribution. An interesting "mesoscopical" approach of displaying microscopical information, that has been obtained with image analysis, at the sample (macroscopical) resolution will be shown. 